UMH-MG-97/03

ULB-Th-97/13

\title{
Chiral supersymmetric pp-wave solutions of IIA supergravity
}

\author{
Cl. Gabriell. 2 , Ph. Spindel目, \\ Mécanique et Gravitation \\ Université de Mons-Hainaut, \\ 15, avenue Maistriau, B-7000 Mons, Belgium \\ M. Rooman日, \\ Physique Théorique, C.P. 225, \\ Université Libre de Bruxelles, \\ bvd du Triomphe, B-1050 Bruxelles, Belgium
}

\begin{abstract}
We describe solutions of type IIA $(\mathrm{N}=2, \mathrm{D}=10)$ supergravity built under the assumption of the existence of at least one residual chiral supersymmetry. Their geometry is of $p p$-wave type. Explicit parametrization of the metric and matter field components, in terms of Killing spinors and arbitrary functions, is provided.
\end{abstract}

\footnotetext{
${ }^{1}$ Aspirant du FNRS

${ }^{2}$ E-mail gabriel@sun1.umh.ac.be

${ }^{3}$ E-mail spindel@sun1.umh.ac..be

${ }^{4}$ Maître de recherches FNRS

${ }^{5}$ E-mail marianne@ucmb.ulb.ac.be
} 


\section{Introduction}

The quest for exact solutions of the supergravity equations is an important task towards our understanding of (the low energy sector of) string theory. A large number of solutions are known today (see for instance [1]). Usually they seem to be based on ansätze imposed on the metric and matter fields, and once solutions are obtained, the existence of residual supersymmetries is checked. Here we shall follow a somewhat different approach, inspired from previous works on supergravity [2, 3]. We shall impose from the beginning that the solutions admit residual supersymmetries and deduce what this assumption implies for the matter fields and metric. Of course, at some point we will have to introduce simplifying assumptions for these fields, but they will be dictated by (the necessary conditions for) the existence of residual supersymmetries.

Our work is organized as follows. We consider type IIA $(\mathrm{N}=2, \mathrm{D}=10)$ supergravity theory, whose bosonic sector consists of the metric tensor, the dilaton field $\phi$, and three Maxwellian fields: the N.S.-N.S. 3-form H and the R.-R. 2- and 4-forms $\mathbf{G}$ and $\mathbf{F}^{\prime}$. First we remind the bosonic field equations and the supersymmetric transformation rules of the gravitino and spin- $\frac{1}{2}$ fields. The latter define the Killing spinors of the theory as the generators of supersymmetries leaving the field configurations of the solution invariant. We will only have to consider the invariance of the fermionic fields, the invariance of the bosonic ones being ensured by imposing the absence of fermionic condensates. We shall establish conditions for the Killing spinor equations to admit at least one solution of a given chirality. This will allow to split the equations into three subsets and show the entirely different rôles played by the N.S.-N.S. field $\mathbf{H}$ and the R.-R. fields $\mathbf{G}$ and $\mathbf{F}^{\prime}$.

In a first stage, we shall assume that $\mathbf{H}=0$. This will imply that the Ricci tensor of the space-time (and as a consequence the energy-momentum tensor) has to be of pure radiation type [see eq. (18)] i.e. entirely characterized by a single lightlike vector $k_{\mu}$. Moreover the gradiant of the dilaton field $\phi$ will appear to be proportional to this vector, thereby implying that it only depends on a single null coordinate $u$. To go ahead, we shall assume that the Maxwellian fields depend also only on $u$, as well as all the metric components but one (otherwise the geometry would inevitably be singular). So, the metric is of $p p$-wave type and all field equations, but one, become algebraic.

Then, we discuss the constraints that the existence of chiral residual supersymmetries impose on the R.-R. field components and give an explicit parametrization of the solution. We also indicate the slight modifications provided by the reintroduction of a non-vanishing N.S.-N.S. field in the previous framework. Finally, we conclude with a few geometric and physical considerations.

Type IIA supergravity is obtained from the $(\mathrm{N}=1, \mathrm{D}=11)$ supergravity theory [4] by a $S^{1}$ dimensional reduction [5, 6, 7]. The idea of looking for conditions for residual supersymmetries have been considered previously in the context of $(\mathrm{N}=1$, $\mathrm{D}=11$ ) supergravity [2, 3]. However, our approach and results are different. Indeed, we start directly from the 10-dimensional theory, which, as will be shown, allows a better control and understanding (at least for us) of the geometric conditions 
imposed on the fields. Moreover, we do not assume a priori our solution to be of the form $\mathcal{M}^{4} \times \mathcal{B}$, where $\mathcal{M}^{4}$ is a maximally 4 -dimensional symmetric space and $\mathcal{B}$ a euclidean internal space. On the other hand, we would also like to mention the work [8] performed in the context of type I supergravity. Its author starts from the assumption of a $p p$-wave geometry and uses different ansätze before looking for residual supersymmetries. He also shows the relevance of such geometries to the full quantum superstring theory.

\section{$2 \quad$ Field equations}

The bosonic field content of type IIA supergravity consists of a 10-dimensional metric tensor $g_{\mu \nu}$, a scalar field $\phi$ and three antisymmetric fields $G_{\mu \nu}, H_{\mu \nu \rho}$ and $F_{\mu \nu \rho \sigma}$ and their potentials. Hereafter we follow the conventions of [5]. The metric signature is $(+,-, \ldots,-)$. The Clifford algebra is defined by $\left\{\mathbb{\Gamma}_{\mu}, \mathbb{\Gamma}_{\nu}\right\}=2 \eta_{\mu \nu}$, where the $\mathbb{\Gamma}$ matrices are $32 \times 32$ and $\mathbb{\Gamma}_{11}=i \mathbb{\Gamma}_{0} \ldots \mathbb{\Gamma}_{9}$. We remind that in $9+1$ dimensions these matrices can be chosen purely imaginary. The Newton constant is set equal to $1 / 4 \pi$.

The bosonic field equations can be written as

$$
\begin{aligned}
R_{\mu \nu}= & \frac{9}{8} \partial_{\mu} \phi \partial_{\nu} \phi-2 \exp \left(\frac{9}{4} \phi\right)\left[G_{\mu \alpha} G_{\nu}{ }^{\alpha}-\frac{1}{16} g_{\mu \nu} G_{\alpha \beta} G^{\alpha \beta}\right] \\
& +\exp \left(-\frac{3}{2} \phi\right)\left[H_{\mu \alpha \beta} H_{\nu}{ }^{\alpha \beta}-\frac{1}{12} g_{\mu \nu} H_{\alpha \beta \gamma} H^{\alpha \beta \gamma}\right] \\
& -\frac{1}{3} \exp \left(\frac{3}{4} \phi\right)\left[F^{\prime}{ }_{\mu \alpha \beta \gamma} F^{\prime \alpha \beta \gamma}-\frac{3}{32} g_{\mu \nu} F^{\prime}{ }_{\alpha \beta \gamma \delta} F^{\alpha \beta \gamma \delta}\right]
\end{aligned}
$$

and

$$
\begin{aligned}
& \nabla_{\alpha}\left[\exp \left(\frac{9}{4} \phi\right) G^{\alpha \mu}\right]=\frac{1}{3} \exp \left(\frac{3}{4} \phi\right) F^{\prime \mu \alpha \beta \gamma} H_{\alpha \beta \gamma} \\
& \nabla_{\alpha}\left[\exp \left(-\frac{3}{2} \phi\right) H^{\alpha \mu \nu}+2 \exp \left(\frac{3}{4} \phi\right) F^{\prime \alpha \mu \nu \beta} A_{\beta}\right]=\frac{1}{576} \frac{\epsilon^{\mu \nu \alpha_{1} \ldots \alpha_{8}}}{\sqrt{-g}} F_{\alpha_{1} \ldots \alpha_{4}} F_{\alpha_{5} \ldots \alpha_{8}} \\
& \nabla_{\alpha}\left[\exp \left(\frac{3}{4} \phi\right) F^{\prime \alpha \mu \nu \rho}\right]=-\frac{1}{72} \frac{\epsilon^{\mu \nu \rho \alpha_{1} \ldots \alpha_{7}}}{\sqrt{-g}} F_{\alpha_{1} \ldots \alpha_{4}} H_{\alpha_{5} \ldots \alpha_{7}} \\
& \square \phi=-\left[\exp \left(\frac{9}{4} \phi\right) G_{\alpha \beta} G^{\alpha \beta}+\frac{2}{9} \exp \left(-\frac{3}{2} \phi\right) H_{\alpha \beta \gamma} H^{\alpha \beta \gamma}+\frac{1}{36} \exp \left(\frac{3}{4} \phi\right) F^{\prime}{ }_{\alpha \beta \gamma \delta} F^{\prime \alpha \beta \gamma \delta}\right]
\end{aligned}
$$

where the 2, 3 and 4-forms $\mathbf{G}, \mathbf{H}$ and $\mathbf{F}$ are closed, while the 4 -form $\mathbf{F}^{\prime}$ is defined as

$$
\mathbf{F}^{\prime}=\mathbf{F}+2 \mathbf{A} \wedge \mathbf{H}
$$

where $\mathbf{A}$ is a potential for $\mathbf{G}(=\mathbf{d A})$ with components $A_{\mu}$. Generators of residual supersymmetries are given by $32-$ component spinors $\eta$, solutions of the equations defining the Killing spinors of the theory:

$$
\nabla_{\mu} \eta+\frac{1}{32} \exp \left(\frac{9}{8} \phi\right) G_{\alpha \beta}\left(\mathbb{\Gamma}_{\mu}^{\alpha \beta}-14 \delta_{\mu}^{\alpha} \mathbb{\Gamma}^{\beta}\right) \mathbb{T}^{11} \eta
$$




$$
\begin{gathered}
+\frac{i}{48} \exp \left(-\frac{3}{4} \phi\right) H_{\alpha \beta \gamma}\left(\mathbb{\Gamma}_{\mu}{ }^{\alpha \beta \gamma}-9 \delta_{\mu}^{\alpha} \mathbb{T}^{\beta \gamma}\right) \mathbb{T}^{11} \eta \\
+\frac{i}{128} \exp \left(\frac{3}{8} \phi\right) F^{\prime}{ }_{\alpha \beta \gamma \delta}\left(\mathbb{T}_{\mu}{ }^{\alpha \beta \gamma \delta}-\frac{20}{3} \delta_{\mu}^{\alpha} \mathbb{\Gamma}^{\beta \gamma \delta}\right) \eta=0
\end{gathered}
$$

that implies the gravitinos' invariance and

$$
\begin{gathered}
\mathbb{\Gamma}^{\mu}\left(\nabla_{\mu} \phi\right) \mathbb{T}^{11} \eta+\frac{1}{2} \exp \left(\frac{9}{8} \phi\right) G_{\alpha \beta} \mathbb{T}^{\alpha \beta} \eta \\
+\frac{i}{9} \exp \left(-\frac{3}{4} \phi\right) H_{\alpha \beta \gamma} \mathbb{\Gamma}^{\alpha \beta \gamma} \eta-\frac{i}{72} \exp \left(\frac{3}{8} \phi\right) F^{\prime}{ }_{\alpha \beta \gamma \delta} \mathbb{T}^{\alpha \beta \gamma \delta} \mathbb{T}^{11} \eta=0
\end{gathered}
$$

that implies the spin- $\frac{1}{2}$ field invariance.

\section{Existence conditions for chiral supersymmetry}

Following the strategy described in the introduction, we impose the chirality condition on the Killing spinor $\eta$ :

$$
i \mathbb{\Gamma}^{11} \eta=\eta \quad
$$

thereby considering at most one half of the supersymmetries. Indeed, this condition limits the number (of positive chirality) residual supersymmetries $N_{r}^{+}$to 16 . Then we project the equations (8,9) onto the positive and negative chirality subspaces. As a consequence, the gravitinos' invariance equation splits into

$$
\nabla_{\mu} \eta+\frac{1}{48} \exp \left(-\frac{3}{4} \phi\right) H_{\alpha \beta \gamma}\left(\mathbb{T}_{\mu}^{\alpha \beta \gamma}-9 \delta_{\mu}^{\alpha} \mathbb{T}^{\beta \gamma}\right) \eta=0
$$

and

$$
\left[4 G_{\alpha \beta}\left(\mathbb{\Gamma}_{\mu}{ }^{\alpha \beta}-14 \delta_{\mu}^{\alpha} \mathbb{\Gamma}^{\beta}\right)-\exp \left(-\frac{3}{4} \phi\right) F^{\prime}{ }_{\alpha \beta \gamma \delta}\left(\mathbb{T}_{\mu}{ }^{\alpha \beta \gamma \delta}-\frac{20}{3} \delta_{\mu}^{\alpha} \mathbb{\Gamma}^{\beta \gamma \delta}\right)\right] \eta=0
$$

The spin- $\frac{1}{2}$ field invariance equation (9) leads to only one additional independent equation:

$$
\left[\mathbb{\Gamma}^{\alpha} \partial_{\alpha} \phi-\frac{1}{9} \exp \left(-\frac{3}{4} \phi\right) H_{\alpha \beta \gamma} \mathbb{\Gamma}^{\alpha \beta \gamma}\right] \eta=0 \quad,
$$

indeed its other chirality projection is equivalent to the contraction of eq. (10) with $\mathbb{T}^{\mu}$.

A glance at these equations shows that they simplify drastically when $\mathbf{H}$, the field coupled to 1 - and 5-branes, vanishes. We shall adopt this assumption in a first stage. Then, eq. (10) implies that $\eta$ is a covariantly constant spinor:

$$
\nabla_{\mu} \eta=0
$$

and eq. (12) that the gradiant of the scalar field $\phi$ is lightlike:

$$
\nabla_{\alpha} \phi \nabla^{\alpha} \phi=0
$$

Moreover, by applying the Dirac operator to eq. (12), we obtain that

$$
\square \phi=0 \quad \text {. }
$$


From the integrability conditions ensuring the existence of the covariantly constant spinor

$$
R_{\mu \nu \alpha \beta} \mathbb{\Gamma}^{\alpha \beta} \eta=0
$$

and from its contraction with $\mathbb{T}^{\nu}$, we deduce that

$$
R_{\mu \alpha} \mathbb{\Gamma}^{\alpha} \eta=0 \quad .
$$

This implies that the Ricci tensor must be of the form

$$
R_{\mu \nu}=r k_{\mu} k_{\nu} \quad,
$$

where the proportionality factor $r$ can be set equal to 1 (owing to the positive energy condition satisfied by the matter energy-momentum tensor). The vector field $k_{\mu}$ is lightlike and the Killing spinor verifies

$$
k_{\alpha} \mathbb{\Gamma}^{\alpha} \eta=0 \quad,
$$

as an immediate consequence of eq. (16). This implies that again one half of the remaining chiral supersymmetries are broken and $N_{r}^{+} \leq 8$. Furthermore, as eq. (12) reduces to $\mathbb{T}^{\alpha} \partial_{\alpha} \phi \eta=0$, we obtain

$$
k_{\mu} \div \partial_{\mu} \phi \quad \text {. }
$$

Hence $k_{\mu}$ is a null, non-twisting, geodesic vector; the latter property results from the Bianchi identities. Moreover eq. (13) implies that the vector field $\bar{\eta} \mathbb{T}^{\mu} \eta$ is also covariantly constant, and eqs (9, 19) that it is proportional to $k^{\mu}$.

\section{Exact solutions}

In order to go ahead, we have to make ansätze on the metric and the remaining fields. The existence of a covariantly constant vector field strongly suggest to consider as space-time geometries the so called plane-fronted gravitational waves with parallel rays ( $p p$-waves)[see ref. [9], chap. 21 and the references therein]. Accordingly, we specialize the metric as

$$
d s^{2}=2 d u\left(d v+A\left[u, x^{a}\right] d u\right)-\sum_{a} \exp \left(2 B_{a}[u]\right)\left(d x^{a}\right)^{2} \quad,
$$

where the index $a$ runs from 1 to 8 . Moreover we suppose that the scalar field is not constant. It can thus be chosen as depending only on the first (null) coordinate, denoted hereafter by $u$. A simple "natural condition" consists of imposing as structure of the skew symmetric tensors

$$
\begin{array}{ll}
\mathbf{G}=d \phi \wedge \mathcal{G}[u] & i\left(\vec{\partial}_{u}\right) \mathcal{G}=0 \\
\mathbf{F}=d \phi \wedge \mathcal{F}[u] & i\left(\vec{\partial}_{u}\right) \mathcal{F}=0
\end{array}
$$

Moreover, to ensure that the Ricci tensor has the structure (18), the trace of the energy-momentum tensor has to vanish according to the Einstein equations. 
Equations (20,22) lead to an expression of the trace that is positive definite, and given by the sum of quadratic contractions of the fields $\mathbf{G}$ and $\mathbf{F}$. In order for the trace to vanish, the 1 - and 3 - forms $\mathcal{G}$ and $\mathcal{F}$ must satisfy:

$$
i\left(\vec{\partial}_{v}\right) \mathcal{G}=0 \quad, \quad i\left(\vec{\partial}_{v}\right) \mathcal{F}=0 \quad .
$$

As a consequence, the quadratic contractions of $\mathbf{G}$ and $\mathbf{F}$ that occur as factors of the metric tensor in the matter energy-momentum tensor [see eq. (1)] and in the scalar field equation (5) vanish individually. Furthermore the only non-identically zero component of the energy-momentum tensor that remains, is $T_{u u}$.

So, the Einstein field equations (1) reduce to $R_{u u}=2 T_{u u}$ i.e.:

$$
\begin{aligned}
& \sum_{a} \exp \left(-2 B_{a}\right) \partial_{x^{a}}^{2} A-\sum_{a} \exp \left(-B_{a}\right) \partial_{u}^{2} \exp \left(B_{a}\right)= \\
& \left(\partial_{u} \phi\right)^{2}\left[\frac{9}{8}-2 \exp \left(\frac{9}{4} \phi\right) \mathcal{G}_{a} \mathcal{G}^{a}-\frac{1}{3} \exp \left(\frac{3}{4} \phi\right) \mathcal{F}_{a b c} \mathcal{F}^{a b c}\right]
\end{aligned}
$$

To cancel the $x^{a}$ dependence of $A$ in this equation, we have to impose that its (flat) Laplacian with respect to the rescaled variables $X^{a}=\exp \left(B_{a}\right) x^{a}$ depends only on $u$ :

$$
\sum_{a} \partial_{X^{a}}^{2} A=h[u]
$$

Hence,

$$
A=\frac{1}{2} h[u]\left(X^{1}\right)^{2}+\mathcal{A}\left[u, X^{a}\right] \quad,
$$

where the function $\mathcal{A}$ is a sum of products of arbitrary $u$-functions by harmonic polynomials of second degree $\boldsymbol{\theta}$ so as to ensuring the finiteness of the Riemann tensor. If we accept space-time singularities, for instance for introducing sources (branes), the function $\mathcal{A}$ can be more general. If it involves harmonic polynomials of degree larger than 2 , it will lead to singularities at spatial infinity. If it involves d-dimensional $(\mathrm{d} \leq 8)$ euclidean Green functions of the Laplacian or their derivative, it will lead to singularities located on 10-d space-time submanifolds [describing shock-waves or (8-d)-branes moving at the speed of light].

Thus, at this stage, we may arbitrarily choose the $u$-dependence of all the functions $B_{a}, \mathcal{A}, \phi, \mathcal{G}_{a}$ and $\mathcal{F}_{a b c}$ and fix the value of $h$ by eqs (24,25):

$$
h=\sum_{a} \exp \left(-B_{a}\right) \partial_{u}^{2} \exp \left(B_{a}\right)+\left(\partial_{u} \phi\right)^{2}\left[\frac{9}{8}-2 \exp \left(\frac{9}{4} \phi\right) \mathcal{G}_{a} \mathcal{G}^{a}-\frac{1}{3} \exp \left(\frac{3}{4} \phi\right) \mathcal{F}_{a b c} \mathcal{F}^{a b c}\right]
$$

Note that, if we assume the metric to be non-singular, the second derivatives of the functions $\exp \left(B_{a}\right)$ have to be non-negative. As a consequence, it is necessary to consider the function $h$ that will be positive, unless all the matter fields vanish and the functions $B_{a}$ are constant. Hence, before imposing the existence of residual supersymmetries, the solution obtained depends on $8+35$ arbitrary functions appearing in the metric (if it is non-singular, otherwise more) and $1+8+56$ in the matter fields. Actually, one of these functions (if not constant) may be set equal to $u$, just by redefining this coordinate.

\footnotetext{
${ }^{6}$ Constant and linear functions can be eliminated by suitable coordinate transformations
} 


\section{$5 \quad$ Residual supersymmetries}

First note that in the coframe defined by $\theta^{\hat{u}}=d u, \theta^{\hat{v}}=(d v+A d u)$, and $\theta^{\hat{a}}=$ $\exp \left(B_{a}\right) d x^{a}$, the only non-vanishing components of the connection 1-form are $\omega_{\hat{u} \hat{a}}=$ $\sum_{a}\left[\exp \left(-B_{a}\right) \partial_{x^{a}} A \theta^{\hat{u}}+\partial_{u} B_{a} \theta^{\hat{a}}\right]$. Hence, owing to eq. (12) that reads now $\mathbb{T}^{u} \eta=$ 0 , the covariant derivatives of $\eta$ reduce to ordinary derivatives and the solutions of eq. (13) are simply given by spinors whose components are constant. In addition, to be a Killing spinor, $\eta$ must still satisfy the algebraic condition :

$$
\left[\mathcal{G}_{a} \mathbb{T}^{a}-\frac{1}{6} \exp \left(-\frac{3}{4} \phi\right) \mathcal{F}_{a b c} \mathbb{T}^{a b c}\right] \eta=0
$$

that results from eq. (11). The simplest way we found to discuss this equation consists of using a representation of the $\mathbb{T}$-matrices built from $8 \times 8$ real euclidean $\Gamma_{\mathrm{i}}$-matrices $(\mathrm{i}=1, \ldots, 7)$ and $\Gamma_{8}=\mathbb{I}_{8}$, the 8-dimensional identity matrix. In this representation, the 32-component spinor $\eta$ is expressed as four copies, with appropriate signs, of an 8-component spinor $\xi$ and eq. (28) reduces to:

$$
\left[\mathcal{G}^{8} \mathbb{I}_{8}+\mathcal{G}^{\mathrm{i}} \Gamma_{\mathrm{i}}-\frac{1}{2} \exp \left(-\frac{3}{4} \phi\right) \mathcal{F}^{8 \mathrm{ij}} \Gamma_{\mathrm{ij}}-\frac{1}{6} \exp \left(-\frac{3}{4} \phi\right) \mathcal{F}^{\mathrm{ij} \mathrm{k}} \Gamma_{\mathrm{ijk}}\right] \xi=0
$$

The rank $(n \leq 8)$ of this linear homogeneous equation fixes the number of residual chiral supersymmetries as $N_{r}^{+}=8-n$. Moreover, each such residual supersymmetry reduces the number of arbitrary matter field functions by 8 . For instance, $N_{r}^{+}=8$ implies that the matter fields $\mathbf{G}$ and $\mathbf{F}$ vanish, whereas $N_{r}^{+}=1$ fixes the components

$$
\begin{aligned}
\mathcal{G}^{8} & =\frac{1}{6} \exp \left(-\frac{3}{4} \phi\right) \mathcal{F}^{\mathrm{ijk}} \bar{\xi} \Gamma_{\mathrm{ijk}} \xi \\
\mathcal{G}^{\mathrm{i}} & =\frac{1}{2} \exp \left(-\frac{3}{4} \phi\right) \mathcal{F}^{8 \mathrm{jk}} \bar{\xi} \Gamma^{\mathrm{i}}{ }_{\mathrm{jk}} \xi+\frac{1}{6} \exp \left(-\frac{3}{4} \phi\right) \mathcal{F}^{\mathrm{jk} \mathrm{l}} \bar{\xi} \Gamma^{\mathrm{i}}{ }_{\mathrm{jkl}} \xi
\end{aligned}
$$

In order to describe the general case, it is useful to introduce an orthonormal basis $\left\{\chi_{I}, I=1, \ldots, 8\right\}$ of the 8 -dimensional spinor space and express the matrix

$$
\mathcal{G}^{8} \mathbb{I}_{8}+\mathcal{G}^{\mathrm{i}} \Gamma_{\mathrm{i}}-\frac{1}{2} \exp \left(-\frac{3}{4} \phi\right) \mathcal{F}^{8 \mathrm{ij}} \Gamma_{\mathrm{ij}}-\frac{1}{6} \exp \left(-\frac{3}{4} \phi\right) \mathcal{F}^{\mathrm{ij} k} \Gamma_{\mathrm{ijk}}
$$

as

$$
P^{[I, J]} \chi_{[I} \bar{\chi}_{J]}+Q^{(I, J)} \chi_{(I} \bar{\chi}_{J)}
$$

The $P^{[I, J]}$ and $Q^{(I, J)}$ coefficients, and the matter field components are related as follows:

$$
\begin{aligned}
P^{[I, J]} & =\mathcal{G}^{\mathrm{i}} \bar{\chi}_{I} \Gamma_{\mathrm{i}} \chi_{J}-\frac{1}{2} \exp \left(-\frac{3}{4} \phi\right) \mathcal{F}^{8 \mathrm{ij}} \bar{\chi}_{I} \Gamma_{\mathrm{ij}} \chi_{J} \\
Q^{(I, J)} & =\mathcal{G}^{8} \delta_{I J}-\frac{1}{6} \exp \left(-\frac{3}{4} \phi\right) \mathcal{F}^{\mathrm{ijk}} \bar{\chi}_{I} \Gamma_{\mathrm{ijk}} \chi_{J} \\
\mathcal{G}^{8} & =\frac{1}{8} \sum_{I} Q^{(I, I)}
\end{aligned}
$$




$$
\begin{aligned}
\mathcal{G}^{\mathrm{i}} & =-\frac{1}{8} \sum_{I, J} P^{[I, J]} \bar{\chi}_{I} \Gamma^{\mathrm{i}} \chi_{J} \\
\exp \left(-\frac{3}{4} \phi\right) \mathcal{F}^{8 \mathrm{ij}} & =\frac{1}{8} \sum_{I, J} P^{[I, J]} \bar{\chi}_{I} \Gamma^{\mathrm{ij}} \chi_{J} \\
\exp \left(-\frac{3}{4} \phi\right) \mathcal{F}^{\mathrm{ijk}} & =\frac{1}{8} \sum_{I, J} Q^{(I, J)} \bar{\chi}_{I} \Gamma^{\mathrm{ij} \mathrm{k}} \chi_{J}
\end{aligned}
$$

Using the spinorial representation (33) of the matrix of the linear system defining the residual supersymmetries, we see that imposing the existence of $N_{r}^{+}$of them implies that

$$
P^{[I, J]}+Q^{(I, J)}=0 \quad \text { for } \quad J=1, \ldots, N_{r}^{+}
$$

in a spinorial basis such that the $N_{r}^{+}$first basis spinors are those defining the residual supersymmetries. In such a basis the solutions of these equations read

$$
\begin{array}{ll}
P^{[K, L]}=0 \quad, \quad Q^{(K, L)}=0 & \text { for } \quad K, L=1, \ldots, N_{r}^{+} \\
Q^{(I, K)}=-P^{[I, K]} & \text { for } \quad K=1, \ldots, N_{r}^{+} \quad, \quad I=1, \ldots, 8
\end{array}
$$

putting into evidence the $8 N_{r}^{+}$constraints imposed by the $N_{r}^{+}$residual supersymmetries. So, by choosing the functions $P^{[I, J]}$ and $Q^{(I, J)}$ according to eqs (41) and an explicit representation of the $8 \times 8 \Gamma$-matrices, we obtain immediately the expression of the matter field components by eqs (36-39). Moreover, the metric function $h(u)$ [eq. (27)] can be expressed in these terms as:

$h=\sum_{a} \exp \left(-B_{a}\right) \partial_{u}^{2} \exp \left(B_{a}\right)+\left(\partial_{u} \phi\right)^{2}\left[\frac{9}{8}+\frac{1}{4} \exp \left(\frac{9}{4} \phi\right) \sum_{I, J}\left[\left(Q^{(I, J)}\right)^{2}+\left(P^{[I, J]}\right)^{2}\right]\right]$

\section{Reintroducing the 3-form field}

In order to simplify the equations, we have imposed almost from the beginning that the matter field $\mathbf{H}$ vanishes. We may however introduce it in the previous framework by imposing that its structure is similar to that of the $\mathbf{G}$ and $\mathbf{F}$ fields [eq. (22)]:

$$
\mathbf{H}=d \phi \wedge \mathcal{H}[u] \quad i\left(\vec{\partial}_{u}\right) \mathcal{H}=0 \quad i\left(\vec{\partial}_{v}\right) \mathcal{H}=0 \quad .
$$

This form ensures that the matter field equations (2) to 5) remain trivially satisfied with the 4-form $\mathbf{F}$ replaced by $\mathbf{F}^{\prime}$, which remains of the form (22), while the energymomentum tensor, except for this last redefinition, changes only by the addition of the extra contribution in $T_{u u}$ :

$$
\left(\partial_{u} \phi\right)^{2} \exp \left(-\frac{3}{2} \phi\right) \mathcal{H}_{a b} \mathcal{H}^{a b}
$$

The residual supersymmetries, which must satisfy eq. (12), are still given by spinors obeying relation (19). Moreover, they continue to depend on the sole coordinate $u$ and must be solution of:

$$
\frac{d \eta}{d u}-\frac{1}{4} \exp \left(-\frac{3}{4} \phi\right) \partial_{u} \phi \mathcal{H}_{a b} \mathbb{\Gamma}^{a b} \eta=0
$$


These spinors can again be expressed in terms of 8-dimensional spinors that have to solve the first order differential system:

$$
\frac{d \xi}{d u}+\Omega \xi=0
$$

where the matrix $\Omega$ is

$$
\Omega=-\frac{1}{4} \exp \left(-\frac{3}{4} \phi\right) \partial_{u} \phi\left(2 \mathcal{H}^{8 \mathrm{i}} \Gamma_{\mathrm{i}}+\mathcal{H}^{\mathrm{ij}} \Gamma_{\mathrm{ij}}\right)
$$

This matrix being antisymmetric, the solutions of eqs (46) can be chosen orthonormed. Actually, starting from an orthonormal basis of the spinor space, and rotating it with an arbitrary $u$-dependent $\mathrm{SO}(8)$ matrix, we obtain 8 orthonormed $u$-dependent spinors that may be used to parametrize the field strength $\mathcal{H}$ from the rotation velocity of the spinorial frame. According to the subset of these solutions that we want to be residual supersymmetries, we obtain the same parametrisation of the $\mathbf{G}$ and $\mathbf{F}$ fields given by eqs (36 to 39) and (41), where now the spinors $\chi_{I}$ are explicitly $u$-dependent.

\section{Conclusions}

We would like to notice several points.

1. If the spinors $\eta$ and $\eta^{\prime}$ are both solutions of eq. (45), the vector $\bar{\eta}^{\prime} \mathbb{T}^{\alpha} \eta$ is a Killing vector, which is covariantly constant if $\mathbf{H}=0$ or $\eta^{\prime}=\eta$. But, due to eq. (19), its only non-vanishing component is $k^{v}$. This explains why the introduction of the metric component $A\left[u, x^{a}\right]$ does not affect the supersymmetries.

2. Here above we have only considered (positive) chiral supersymetry generators. Of course, this did not exclude a priori the existence of other supersymmetries generated by generators of negative chirality or non-chiral generators. For instance, it is obvious that if the R..-R. fields vanish, there exists in addition to the 8 supersymmetries described in the text, 8 other supersymmetries of opposite chirality. More generally, when some combinations of the components of the Maxwellian fields vanish, extra supersymmetries with negative chirality appear. For example, it will be the case if $\mathcal{H}=0$ and only the $\mathcal{G}^{8}$ and $\mathcal{F}^{8 i j}$ components of $\mathcal{G}$ and $\mathcal{F}$ are non-zero, or if $\mathcal{H}=0$, and only $\mathcal{G}^{\mathrm{i}}$ and $\mathcal{F}^{\mathrm{ijk}}$ are non-zero.

3. It is worthwhile to notice that our basic assumption of the existence of a chiral residual supersymmetry "naturally" leads to $p p$-wave geometries whose quantum properties are quite remarkable: positive and negative frequency modes are well defined on them, and they constitute solutions of the effective field theory of superstring theories to all orders in the string tension parameter [8]. 
4. The main property of the solutions we obtain is that they depend on a huge number of arbitrary functions. From a geometric point of vue, they correspond to $p p$-waves. Physically, they represent waves traveling along null curves generated by the (covariantly constant) vector $k_{\mu}$; the various arbitrary functions occurring in the solution describe the profiles of the waves. An apparent weakness of such solutions is that their energy is infinite, except if the appropriate spatial coordinate is compactified. Nevertheless, they can be considered as approximation of radiation fields (which could have been emitted by radiating branes?) propagating in a given direction, on a general background [10].

5. As emphasized by F. Englert [11], the Aichelburg-Sexl metric [12] is of the type discussed here. Accordingly, in 10 dimensions, the metric describing the gravitational field of a black-hole moving at the speed of light admits 16 residual supersymmetries, which will be maintained if we reduce this geometry to 4 dimensions. This is to be put in relation with the existence of short massless supersymmetric multiplets.

We are grateful to R. Argurio, F. Englert and L. Houart for useful conversations.

\section{References}

[1] J. M. Maldacena, Black holes in string theory, Ph. D. thesis (1996) Princeton University, hep-th/9607235

[2] P. Candelas, D. J. Raine, Nucl. Phys. B 248 (1984) 415

[3] B. de Wit, D. J. Smit, N. D. Hari Dass, Nucl. Phys. B 283 (1987) 165

[4] E. Cremmer, B. Julia, Nucl. Phys. B 159 (1979) 141

[5] I.C.G. Campbell, P.C. West, Nucl. Phys. B 243 (1984) 112

[6] M. Huq, M. A. Namazie, Class. \& Quant. Grav. 2 (1985) 293, Class. \& Quant. Grav. 2 (1985) 597

[7] M. Pernici, F. Gianni, Phys. Rev. D 30 (1984) 29

[8] R. Güven, Phys. Lett.B 191 (1987) 275

[9] D. Kramer, H. Stephani, E. Herlt, M. MacCallum, Exact solutions of Einstein's field equations (1979) Cambridge Univ. Press

[10] R. Penrose, in Differential Geometry and Relativity, eds M. Cahen, M. Flato (1976) D. Reidel Pub. Cie, Dordrecht, Holland, page 271

[11] F. Englert, Private communication

[12] P.C. Aichelburg, R.U. Sexl, G.R.G. 2 (1971) 303 